\title{
Utilizing Semantics in the Production of iTV Shows
}

\author{
Georg Güntner, Dietmar Glachs, and Rupert Westenthaler \\ Salzburg Research Forschungsgesellschaft m.b.H. \\ Jakob Haringer Straße 5/III, A-5020 Salzburg, Austria \\ \{georg.guentner, dietmar.glachs, \\ rupert.westenthaler\} @salzburgresearch.at
}

\begin{abstract}
This paper gives an overview of the semantic aspects of an advanced, semantics-based broadcasting production support system designed to enable the creation of interactive multi-channel television shows. The "Intelligent Media Framework" forms the middleware of this system and was developed in the context of the European integrated project LIVE. The envisaged "intelligence" is based on formal, machine understandable descriptions of the content and the events. We demonstrate the successful usage of the system by a broadcasting corporation in a field trial with several hundred end consumers, conducted during the Olympic Games in Beijing, August 2008.
\end{abstract}

\section{Introduction}

The European integrated project "LIVE Staging of Media Events" (LIVE, www.liveist.org) aims at the creation of novel intelligent content production methods and tools for broadcasters to stage live media events such as the Olympic Games 2008 in Beijing. In the terminology of the project, "staging live media events" is a notion for the real-time creation of non-linear multi-channel video shows, whose format is adaptive according to the interests of the consumers. While the development of the envisaged interactive content formats forms a research strand of its own [1] the concept also imposes several conceptual and technical challenges from a software engineering and semantic modelling point of view. Fig. 1 depicts the environment the LIVE production support system needs to work in to support the preparation and production of shows as envisioned above.

Staging of media events in the novel TV format comprises all knowledge of an event e.g. the schedule and participants; knowledge of all related information about the event including archived content and - foremost - a detailed knowledge of how the staged event is to be presented to the targeted consumer audience. Typically such information is collected during the preparation phase of a show. However, the LIVE production support system addresses not only the preparation, but also the real time staging of media events where archival knowledge related to the event must be combined with real-time information of what is actually happening during the event. Furthermore, the actual production is influenced by accurate, real-time feedback from the viewing audience. An overview of the preparation and staging workflow is also shown in Fig. 1 which shows the activities of the professional users in their dedicated user spaces. 
The remainder of this paper is structured as follows: Section 2 provides details of the LIVE production support system used for this demonstration; Section 3 outlines how the Intelligent Media Framework (IMF) uses semantic technologies to support knowledge and information integration in the LIVE staging domain.

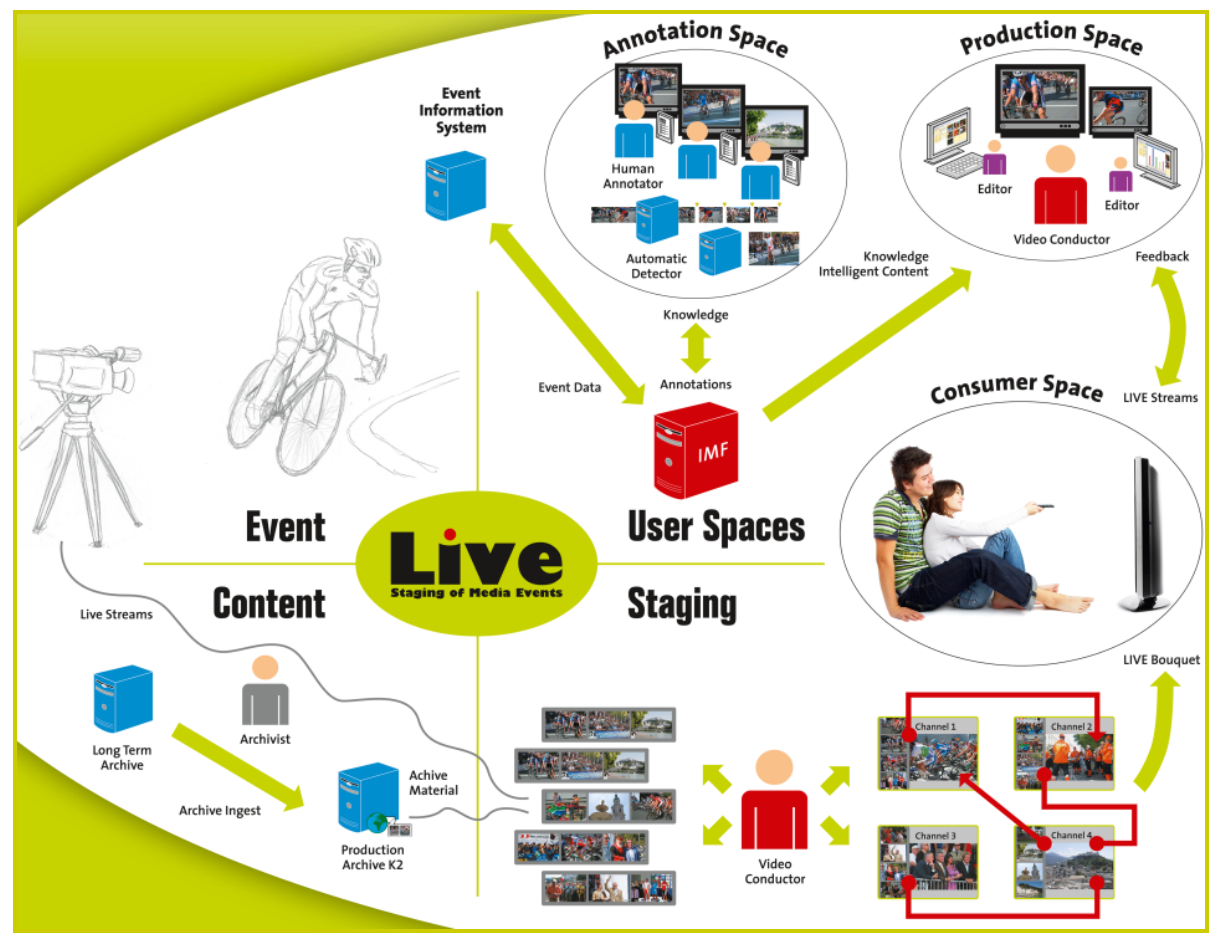

Fig. 1. Overview on the LIVE staging environment

\section{Showcasing the LIVE Production Support System}

Our demonstrator is based on the LIVE production support system used in a field trial during the Summer Olympic Games in August 2008. Over 500 households participated in the iTV trial and gave explicit (e.g. voting) and implicit (e.g. channel switching) feedback to the production team. At the production site in Vienna at ORF, the Austrian Broadcasting Corporation, annotators manually annotated up to twelve live video feeds from the Olympic Games. ATOS origin, the supplier for the Olympic Sports Information System provided event information in real time. This resulted in about 12.000 messages per hour that were sent to the Intelligent Media Framework (IMF). The IMF had to interpret the messages, align them to the knowledge model, maintain the semantic index and propagate a filtered set of messages to the consuming systems (e.g. the recommender system). A dedicated application called "Staging Console" displayed a set of messages filtered according to their relevance for the production process (e.g. priority, schedule). 
The demonstrator outlines the complete information flow by starting with the captured sport event and by simulating the information created by the Olympic Sport Information System. The "Staging Console" as shown in Fig. 2, visualizes the scenery by outlining the observed live feeds (1), the scheduled events presented along a time line (2) and as a schedule of upcoming events (3). It further shows manually and automatically generated messages to the production team (4). Optionally, details of each message can be retrieved in a dedicated panel (5).

Besides the "Staging Console", the "Recommender Application" (see [3]) is part of the LIVE system and allows the production team to easily retrieve video clips from the production archive according to the context of the show. The "Feedback Panel" shows the viewers' channel switching behaviour (implicit feedback) and polling results (explicit user feedback). In the field trial the feedback was collected via the back channel of the set-top boxes of the used IPTV infrastructure (aon.tv ${ }^{1}$ ).

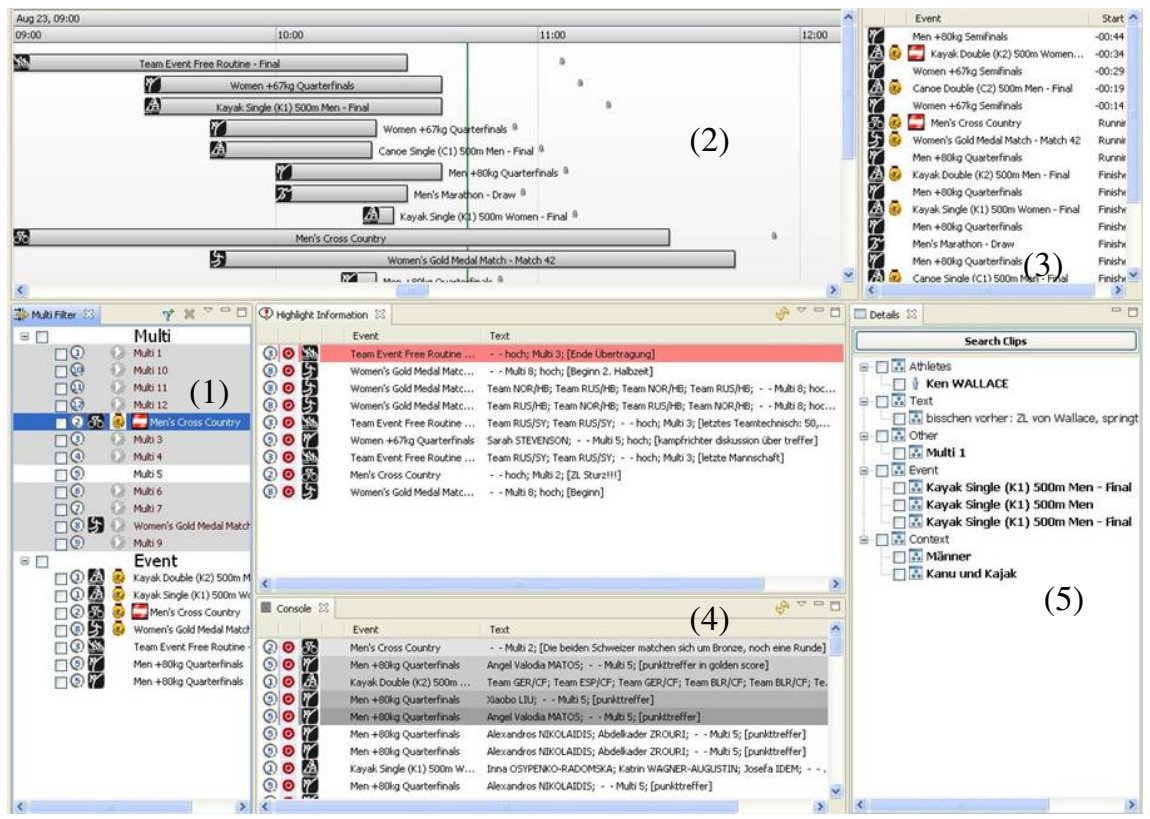

Fig. 2. LIVE Staging Console

The demonstration is a time shifted replay based on data gathered during the Olympic field trial. The demonstration shows the usage of the different tools in the workflow starting with the live capture of video streams, showing the semantic annotation and the bundling of streams into "shows" by the so called "video conductor" who can also react to consumer feedback and change the broadcast accordingly.

\footnotetext{
${ }^{1}$ aon.tv - see http://www.aon.tv/ (in German) - last visited 06.03.2009.
} 


\section{Intelligent Media Framework}

The Intelligent Media Framework (IMF) implements the knowledge based middleware component of the LIVE production support system. This section gives first an overview of the knowledge model used and then describes the principal architecture of the LIVE production support system.

The IMF is based on a knowledge structure divided into three sub-models: the Term Model, The Event Domain Model and the Intelligent Content Model. The Term Model forms the foundation of this knowledge structure and is based on functionalities defined in the Simple Knowledge Organization System (SKOS). It supports the features of SKOS, but in addition it defines a base typology for terms, the "Term Type Hierarchy". This typology aims to simplify the structuring of the controlled vocabulary and provides additional semantics to the other knowledge models of the IMF. All terms in the controlled vocabularies are aligned to the Term Type Hierarchy. Details of the entire knowledge model can be found in [2].

On top of the Term Model is the Event Domain Model. This model supports the definition of formal statements describing the current state (annotating) or a future state (planning) of events. Such statements are used to describe the staged event as well as the staging process itself. They are instantiations of concepts defined in the Event Domain Model (e.g. Agent, Activities, Events, Settings). Terms and part of the controlled vocabulary are used to parameterize such statements.

The Intelligent Content Model defines knowledge-based content objects called Intelligent Media Assets (IMAs) which manage the relation between content and the knowledge. To achieve this, an IMA consists of two main parts: (i) Content annotations define knowledge about features of the essence such as storage location(s), encoding, access rights or publication rights. Dedicated elements of existing standards such as MPEG-7 and NewsML are used for such annotations. (ii) Subject matter annotations define information items expressing formal descriptions of what the essence is about. Subject matter descriptions include a spatial-, temporal-, topic based (includes terms as well as instances of the event domain model) and free text classification. In addition they hold information about the creator (professional user/software agent and user role) to support collaborative filtering.

The Intelligent Media Framework provides the knowledge infrastructure for the LIVE production support system and is based on a multi-tier service-oriented architecture in which all interfaces to external components are modeled as Web Services. The service interfaces are structured along the three parts of the knowledge model and provide access to the controlled vocabulary, the event domain model and the intelligent media assets. In addition to these pull-type services, the IMF uses a messaging system to support the real-time aspect of the staging process. Asynchronous messaging is used to process information from internal and external sources (automatic annotators, human annotator and information sent by the event information system). The IMF aligns the information to the already available knowledge of the event as well as the show and propagates the resulting knowledge by publishing it to subscribing applications of the LIVE production support system. This information enrichment uses statements as defined by the Event Domain model to formally describe the current state of the event as well as the staged show. 
The IMF also maintains a semantic index over the whole knowledge base. This semantic index uses the relations defines in the Event Domain- and the Term Model to provide tagging-like classifications for all resources. Real-time changes to the knowledge structure are reflected in the semantic index.

During the Olympic Trial the IMF processed around 20.000 terms, around 100.000 semantic relations between the terms; 120.000 event participations and about 100.000 statements describing specific situations in the various events. Around 20.000 Intelligent Media Assets were created during the Olympic Field Trial.

\section{Conclusion}

In the field trial, content-based information was combined with event information in real-time, using a common knowledge model. The combination of content management systems with event information systems was successfully tested. The LIVE production support system demonstrated how a knowledge based system can enable knowledge workers to create the envisioned LIVE iTV format: Editors and directors at the production site at ORF, the Austrian Broadcasting Corporation, confirm that it would not be feasible to create such non-linear multi-channel video shows by using traditional production methods.

A survey by GfK Austria showed that the quality of the new format was rated with 1,5 (out of 5 with " 1 " expressing highest satisfaction) by the viewers. $82 \%$ of them expressed a high interest in a continuation of the interactive multi-channel format.

The LIVE approach differs from related research primarily with respect to the real time nature of the production methods (see [4] for a comparable approach in the area of non-linear, yet non real time narrative structures).

\section{Acknowledgments}

The LIVE project ("Live Staging of Media Events") is an integrated project partially funded by the European Commission within the 6th Framework of the IST under grant number FP6-27312. All statements in this work reflect the personal ideas and opinions of the authors and not necessarily the opinions of the European Commission.

\section{References}

1. Grünvogel, S.M., Wages, R., Bürger, T., Zaletelj, J.: A Novel System for Interactive Live TV. In: Ma, L., Rauterberg, M., Nakatsu, R. (eds.) ICEC 2007. LNCS, vol. 4740, pp. 193 204. Springer, Heidelberg (2007)

2. Güntner, G., Bürger, T., Westenthaler, R., Glachs, D.: Basic Specification of the Intelligent Media Framework: Public Synopsis. LIVE Deliverable D7.4 (2007),

http: / /www.ist-live.org

3. Zaletelj, J.: Recommender System for the Multi-channel TV Production. In: Tscheligi, M., Obrist, M., Lugmayr, A. (eds.) EuroITV 2008. LNCS, vol. 5066, pp. 102-106. Springer, Heidelberg (2008)

4. Zsombori, V., Ursu, M., Wyver, J., Kegel, I., Williams, D.: ShapeShifting Documentary: A Golden Age. In: Tscheligi, M., Obrist, M., Lugmayr, A. (eds.) EuroITV 2008. LNCS, vol. 5066, pp. 40-50. Springer, Heidelberg (2008) 